\title{
Stabilization for a class of minimum phase hybrid systems under an average dwell-time constraint
}

\author{
A. R. Teel ${ }^{1}$ and L. Marconi ${ }^{2, *, \dagger}$ \\ ${ }^{1}$ ECE Department, University of California, Santa Barbara, CA, U.S.A. \\ ${ }^{2}$ C.A.SY.-D.E.I.S., University of Bologna, Bologna, Italy
}

\begin{abstract}
SUMMARY
We consider the class of nonlinear systems in normal form with unitary relative degree whose zero and output dynamics are affected by state jumps fulfilling an average dwell-time constraint. Under a minimum-phase assumption requiring the existence of a compact set, which is globally pre-asymptotically stable for the hybrid zero dynamics, we show how to design continuous, global state- and semiglobal output-feedback control laws. The proposed design methodology extends to the considered class of hybrid systems well-known design techniques for robustly stabilizing the class of continuous-time minimumphase nonlinear systems having unitary relative degree. Examples are given to show the usefulness of the technical result. Copyright (C) 2010 John Wiley \& Sons, Ltd.
\end{abstract}

Received 20 October 2009; Revised 22 March 2010; Accepted 14 June 2010

KEY WORDS: hybrid systems; global stabilization; minimum-phase

\section{INTRODUCTION}

The study of dynamical systems combining continuous-time and discrete-time behaviors, usually referred to as hybrid systems, has attracted a lot of attention in the control community due to the fact that a large variety of physical systems can be naturally modeled in this way and due to a number of intriguing theoretical issues that arise when studying this class of systems. The hybrid system literature is indeed very vast and a number of research fields focused on specific classes of hybrid systems can be identified. Besides others, it is worth mentioning the field of switching systems [1, 2], hybrid automata [3], sampled-data systems [4], event-based systems [5] and others. Of course the challenges behind the study of hybrid systems become even more noteworthy if both the continuous- and discrete-time dynamics are nonlinear systems and if uncertainties, such as unknown parameters and/or exogenous disturbances, enter into the picture. In this respect a noteworthy research attempt has been recently done (see the tutorial [6] and the references therein) in order to develop a general framework of hybrid nonlinear systems within which classical stability results, which are known to hold in the continuous- and discrete-time domain, are recovered. In particular, crucial properties linked to robustness of asymptotic stability to data perturbation, representing building blocks of many robust design techniques developed so far in a non-hybrid context, have been clearly shown to also exist in a hybrid nonlinear setting. These developments have made it possible to extend to hybrid systems a number of tools, for analysis and synthesis, commonly used in the non-hybrid literature and, in turn, to provide the theoretical substrate on

\footnotetext{
${ }^{*}$ Correspondence to: L. Marconi, C.A.SY.-D.E.I.S., University of Bologna, Bologna, Italy.

†E-mail: lorenzo.marconi@unibo.it 
which a number of design techniques for robustly controlling hybrid nonlinear systems have been developed and proved to be effective in a rigorous way.

The current work fits in this context as it aims to develop a design tool in the hybrid framework described in [6] by extending a technique commonly used for robust stabilization of nonlinear continuous-time systems. Specifically, one of the cornerstones in the literature about robust stabilization of continuous-time nonlinear systems in the large is represented by [7], in which it is shown how the class of nonlinear systems described in normal form and fulfilling a minimum-phase assumption can be globally stabilized by means of a continuous state-feedback control law. In [7] the minimum-phase assumption is formulated by requiring that the zero dynamics of the system, a notion proposed by the authors of [7] in earlier contributions (see [8,9]), possess a globally asymptotically stable equilibrium point. Among the number of developments inspired by [7], a notable role is played by the results in [10] in which it is shown that partial state feedback (namely a feedback depending only on the output and its time derivatives) succeeds if just stabilization on compact sets (i.e. semi-global stabilization) is sought. In particular, if attention is restricted to nonlinear systems having unitary relative degree, a pure algebraic output feedback has been proved to succeed in stabilizing systems on compacta. Furthermore, the proposed control law can be shown to be effective even in the presence of possible uncertainties affecting the zero and the output dynamics. This, in turn, makes the mentioned result (and other developments proposed in [10]) fundamental as providing systematic tool for robustly stabilizing nonlinear systems by means of continuous output feedback.

The goal of this work is to extend the mentioned (global and semi-global) stabilization tool to a fairly general class of hybrid nonlinear systems having relative degree one and fulfilling a generalized minimum-phase condition. According to the framework of [6], the considered hybrid system is given by the combination of flow dynamics, represented by a nonlinear system in normal form with unitary relative degree, and jump maps that are represented by nonlinear functions of the state. In the framework considered in this paper, the conditions that allow the state of the system to flow, according to the flow dynamics, or to jump, according to the jump maps, are given in terms of flow and jump sets, to which the state of the system must belong for having, respectively, flow or jump enabled, and of an auxiliary clock variable whose role is to enable possible jumps provided that an average dwell time is guaranteed from the previous reset. The concept of average dwell time, originally introduced in [11], has been extensively used in the literature of switching systems in order to frame situations in which the average interval between consecutive switches has a guaranteed lower bound even though multiple switches, not separated by a guaranteed dwell time, may occasionally happen. This concept, representing the natural generalization of the notion of dwell time introduced in [12] in the study of supervisory control, has been discovered interesting in the stability analysis of switching linear and nonlinear systems. In [11] the authors show that switching among Hurwitz linear systems results in a uniformly exponentially stable system provided that switching is 'slow-on-the-average'. The result is extended in [13] in which it is shown that exponential stability is preserved even though the switching involves both Hurwitz and anti-stable linear systems provided that the activation time of the latter is relatively small compared with one of the Hurwitz systems and the overall average dwell time is large enough. The relevance of the notion of average dwell-time in the stability analysis of nonlinear systems subject to reset and in the presence of exogenous signals is also highlighted in [14]. In this work it is shown how input-to-state stability with respect to exogenous disturbances is guaranteed if possible 'stabilizing resets' happen sufficiently frequently in the average and, conversely, possible 'de-stabilizing resets' rarely happen in the average. Recently, in [15], the notion of average dwelltime has been shown to be effective also to study stability of a (possibly infinite-dimensional) family of nonlinear vector fields. In the paper it is shown that any possible switching scenario among the family results in trajectories that are stable in a proper sense (see [15]) provided that each vector field satisfies appropriate asymptotic (not necessarily exponential) stability conditions and that the average dwell time is sufficiently large.

Motivated by the previous and others results, we thus focus on hybrid systems in which the time intervals between consecutive jumps of the state are lower bounded in the average. In this 
context we formulate a minimum-phase assumption requiring that the hybrid dynamics obtained by zeroing the output of the system admit a pre-asymptotically stable (see [16]) compact set and we show how this, in addition to others technical assumptions, suffice to design a continuous, state feedback globally stabilizing the zero dynamics to the compact set and the output to zero for the overall system. As a corollary of this general result we show that pure output feedback succeeds if just semiglobal stability results are sought.

The paper is organized as follows. In the next section we briefly present a few basic notions regarding hybrid systems that are instrumental for the analysis carried out in the paper. Section 3 presents the framework and the main results about global state-feedback and semi-global outputfeedback stabilization. Then, in Section 4, a few examples are presented and discussed. Section 5 concludes with final remarks and future developments.

\section{Notations}

In the paper $\mathbb{R}, \mathbb{R}_{>0}$, and $\mathbb{R}_{\geqslant 0}$ denote respectively the set of real, positive real, and nonnegative real numbers. The symbol $\mathbb{N}$ denotes the set of nonnegative integers. With $\mathbb{R}^{n}$ we indicate the $n$-dimensional Euclidean space. For $x \in \mathbb{R}^{n}$ and $\mathscr{A}$ a set of $\mathbb{R}^{n},|x|_{\mathscr{A}}$ denotes the distance of $x$ from $\mathscr{A}$ defined as $\inf _{y \in \mathscr{A}}|x-y|$. With $F: \mathbb{R}^{m} \rightrightarrows \mathbb{R}^{n}$ we indicate a set-valued mapping from $\mathbb{R}^{m}$ to $\mathbb{R}^{n}$.

\section{PRELIMINARIES}

In this part we briefly review basic notations and fundamental facts about hybrid systems that are instrumental for the analysis of the next section. Following [6], an hybrid system is specified by the data $(F, G, C, D)$ where $F: \mathbb{R}^{n} \rightrightarrows \mathbb{R}^{n}$ and $G: \mathbb{R}^{n} \rightrightarrows \mathbb{R}^{n}$ are set-valued mappings called respectively the flow and the jump map, while $C$ and $D$ are closed subsets of $\mathbb{R}^{n}$ called respectively the flow and the jump set. The mappings $F$ and $G$ are assumed outer semi-continuous and locally bounded and $F(x)$ and $G(x)$ are not empty with $F(x)$ convex for all $x \in C$. Denoting by $x$ the state of the hybrid system, the dynamics underlying $(F, G, C, D)$ is explicitly described by the following set of differential and algebraic inclusions

$$
\begin{gathered}
\dot{x} \in F(x) \quad x \in C \\
x^{+} \in G(x) \quad x \in D
\end{gathered}
$$

where $x^{+}$denotes the next value of the state $x$. As shown in [6], a number of control scenarios commonly referred to as hybrid systems, such as hybrid automata, switching systems, sampled data systems, and others, can be cast in the form (1).

According to [17] the solutions of system (1) can be conveniently defined on hybrid time domains. Specifically, a set $S \subset \mathbb{R} \geqslant 0 \times \mathbb{N}$ is called a hybrid time domain if it is the union of infinitely many interval of the form $\left[t_{j}, t_{j+1}\right] \times\{j\}$, where $0=t_{0} \leqslant t_{1} \leqslant t_{2} \leqslant \ldots$, or of finitely many such intervals, with the last one possibly of the form $\left[t_{j}, t_{j+1}\right] \times\{j\},\left[t_{j}, t_{j+1}\right) \times\{j\}$ or $\left[t_{j}, \infty\right) \times\{j\}$. A solution of (1) is defined in terms of hybrid arc consisting of a hybrid time domain dom $\phi$ and a function $\phi: \operatorname{dom} \phi \rightarrow \mathbb{R}^{n}$ such that $t \mapsto \phi(t, j)$ is locally absolutely continuous for each $j$ such that $(s, j) \in \operatorname{dom} \phi$ for some $s \in \mathbb{R}_{\geqslant 0}$. Specifically, a solution of (1) is represented by a hybrid arc such that $\phi(0,0) \in C \cup D$ and

- for all $j \in \mathbb{N}$ and almost all $t$ such that $(t, j) \in \operatorname{dom} \phi$,

$$
\phi(t, j) \in C \quad \dot{\phi}(t, j) \in F(\phi(t, j))
$$

- for all $(t, j) \in \operatorname{dom} \phi$ such that $(t, j+1) \in \operatorname{dom} \phi$,

$$
\phi(t, j) \in D \quad \phi(t, j+1) \in G(\phi(t, j)) .
$$

In this framework a notion of pre-asymptotic stability has been introduced in [16] as generalization of the classical notion of asymptotic stability to the setting where completeness or even 
existence of solutions are not guaranteed. Specifically, for a compact set $\mathscr{A} \subset \mathbb{R}^{n}$, we have the following definitions (see [16]):

- $\mathscr{A}$ is pre-stable for (1) if for each $\varepsilon>0$ there exists $\delta>0$ such that any solution $\phi$ of (1) with $|\phi(0,0)|_{\mathscr{A}} \leqslant \delta$ satisfies $|\phi(t, j)|_{\mathscr{A}} \leqslant \varepsilon$ for all $(t, j) \in \operatorname{dom} \phi$;

- $\mathscr{A}$ is pre-attractive for (1) if there exists $\delta>0$ such that any solution $\phi$ of (1) with $|\phi(0,0)|_{\mathscr{A}} \leqslant \delta$ is bounded and, if it is complete, $\phi(t, j) \rightarrow \mathscr{A}$ as $t+j \rightarrow \infty$;

- $\mathscr{A}$ is pre-asymptotically stable if it is both pre-stable and pre-attractive.

Furthermore, the set of all $x \in \mathbb{R}^{n}$ from which all the solutions are bounded and the complete ones converge to $\mathscr{A}$ is called pre-domain of attraction. We say that a set $\mathscr{A}$ is globally pre-asymptotically stable if it is pre-asymptotically stable with a pre-domain of attraction equal to $\mathbb{R}^{n}$. Clearly, in case of completeness of solutions, the previous definitions boil down to classical stability notions. We refer the reader to [16] (see also [6]) for a broad discussion about the advantages of the notion of pre-asymptotic stability over 'simple' asymptotic stability and about its relevance in the hybrid context. For the purpose of this paper, it is important to mention that pre-asymptotic stability is equivalent to the existence of a smooth Lyapunov function. In particular, the following converse Lyapunov theorem has been proved in [16] under the assumptions on $(C, F, D, G)$ stated at the beginning of this section.

\section{Theorem 1}

Let $\mathscr{A} \subset \mathbb{R}^{n}$ be a compact set that is globally pre-asymptotically stable for (1). Then, there exists class- $\mathscr{K}_{\infty}$ functions $\alpha_{1}$ and $\alpha_{2}$ and a smooth Lyapunov function $V: \mathbb{R}^{n} \rightarrow \mathbb{R} \geqslant 0$ such that

$$
\alpha_{1}\left(|x|_{\mathscr{A}}\right) \leqslant V(x) \leqslant \alpha_{2}\left(|x|_{\mathscr{A}}\right) \quad \forall x \in \mathbb{R}^{n}
$$

and

$$
\begin{array}{r}
\max _{f \in F(x)}\langle\nabla V(x), f\rangle \leqslant-V(x) \quad \forall x \in C \\
\max _{g \in G(x)} V(g) \leqslant \frac{V(x)}{2} \quad \forall x \in D .
\end{array}
$$

In order to avoid cumbersome terminology, in the following part of the paper we will drop the prefix 'pre' from the above stability notions. Note, however, that completeness and local existence of solutions are not required in the considered stability framework.

\section{MAIN RESULT}

In this paper we consider the hybrid control system with state $\left(\tau, x_{1}, x_{2}\right) \in \mathbb{R} \times \mathbb{R}^{n} \times \mathbb{R}$ satisfying

$$
\left.\begin{array}{rl}
\dot{\tau} & \in[0, \delta] \\
\dot{x}_{1} & =f_{1}\left(\tau, x_{1}, x_{2}\right) \\
\dot{x}_{2} & =f_{2}\left(\tau, x_{1}, x_{2}\right)+b\left(\tau, x_{1}, x_{2}\right) u
\end{array}\right\} \quad\left(\tau, x_{1}, x_{2}\right) \in[0, N] \times C \times \mathbb{R}
$$

where $\delta>0$ and $N \geqslant 1$. The flow dynamics of (2) exhibit a structure that is reminiscent of the normal form with unitary relative degree in the continuous-time setting (see [18]) with the state variables $x_{2}$ and $\left(\tau, x_{1}\right)$ playing the role of output and internal states, respectively. Furthermore, a distinctive feature of (2) is the presence of the variable $\tau$, which has a key role in enabling possible 
jumps. In particular, the specific flow and jump rules underlying the variable $\tau$ lead to conclude that the hybrid system (2) satisfies an average dwell-time as detailed in the next proposition.

\section{Proposition 1}

For each solution $\phi$ of (2) and each $(s, i),(t, j) \in \operatorname{dom} \phi$ with $s+i \leqslant t+j$, the following relation holds

$$
j-i \leqslant \delta(t-s)+N
$$

\section{Proof}

Let $\phi, t \geqslant s, j \geqslant i, N \geqslant 1$ and $\delta>0$ be given. By using the flow and jump rule of the variable $\tau$, it turns out that

$$
0 \leqslant \tau(t, j) \leqslant \tau(s, i)+\delta(t-s)-(j-i) \leqslant N+\delta(t-s)-(j-i) .
$$

The previous expression, properly rearranged, yields the result.

By following the terminology proposed in [11], the number $N$ represents the chatter bound as it denotes the maximum number of consecutive jumps all occurring at the same time while $1 / \delta$ represents the average dwell-time. As a particular case, taking $\delta=1 / \tau_{\max }$ and $N=1$ generates solutions that satisfy a dwell-time constraint on jumps, i.e. all consecutive jumps are separated by at least $\tau_{\max }$ units of time. Further restricting attention to solutions that satisfy $\dot{\tau}=\delta$ generates solutions with periodic jumps with period $\tau_{\max }$.

We will show that system (2) can be globally stabilized by means of a continuous state feedback under the following assumption.

\section{Assumption 1}

The following conditions hold:

1. The sets $C, D \subset \mathbb{R}^{n}$ are closed and the functions $f_{1}, f_{2}, g_{1}, g_{2}$ and $b$ are continuous.

2. For each compact set $K \subset \mathbb{R}^{n} \times \mathbb{R}$ there exists $L>0$ such that

$$
\left|f_{1}\left(\tau, x_{1}, x_{2}\right)-f_{1}\left(\tau, x_{1}, 0\right)\right| \leqslant L\left|x_{2}\right| \quad \forall\left(\tau, x_{1}, x_{2}\right) \in[0, N] \times(K \cap(C \times \mathbb{R})),
$$

and, for all $\left(\tau, x_{1}, x_{2}\right) \in[1, N] \times(K \cap(D \times \mathbb{R}))$,

$$
\max \left\{\left|g_{2}\left(\tau, x_{1}, x_{2}\right)\right|,\left|g_{1}\left(\tau, x_{1}, x_{2}\right)-g_{1}\left(\tau, x_{1}, 0\right)\right|\right\} \leqslant L\left|x_{2}\right| .
$$

3. There exists a nonincreasing continuous function $\underline{b}: \mathbb{R}_{\geqslant 0} \rightarrow \mathbb{R}_{>0}$ such that

$$
b\left(\tau, x_{1}, x_{2}\right) \geqslant \underline{b}\left(\left|\left(x_{1}, x_{2}\right)\right|\right) \quad \forall\left(\tau, x_{1}, x_{2}\right) \in[0, N] \times C .
$$

4. The compact set $[0, N] \times \mathscr{A}$ is globally asymptotically stable for the system

$$
\begin{gathered}
\left.\begin{array}{c}
\dot{\tau} \in[0, \delta] \\
\dot{x}_{1}=f_{1}\left(\tau, x_{1}, 0\right)
\end{array}\right\} \quad\left(\tau, x_{1}\right) \in[0, N] \times C \\
\left.\begin{array}{l}
\tau^{+}=\tau-1 \\
x_{1}^{+}=g_{1}\left(\tau, x_{1}, 0\right)
\end{array}\right\} \quad\left(\tau, x_{1}\right) \in[1, N] \times D .
\end{gathered}
$$

5. The function $f_{2}$ satisfies $f_{2}\left(\tau, x_{1}, 0\right)=0$ for all $\left(\tau, x_{1}\right) \in[0, N] \times \mathscr{A}$.

Item 1 is sufficient to guarantee the (mild) regularity conditions that are required for hybrid system to enjoy a number of properties, including the converse Lyapunov results in [16]. Item 2 asks that the functions $f_{1}$ and $g_{1}, g_{2}$ satisfy a certain type of sector growth condition, respectively on the flow and jump set, with respect to the variable $x_{2}$. The latter assumption is instrumental for the Lyapunov construction characterizing the proof of our main result, given in Theorem 2 below. Item 3 , on the other hand, asks that the function $b$ multiplying the input is bounded from below by a continuous positive function. In this respect, by inheriting the terminology used in 
the continuous-time setting, the function $b$ plays the role of 'high-frequency' gain of the hybrid system and system (2) can be well regarded as a system with unitary relative degree. By insisting on the parallel with the continuous-time setting, system (3) in item 4 represents the zero-dynamics of system (2) and the requirement about global asymptotic stability of the set $\mathscr{A}$ for (3) can be interpreted as a minimum-phase assumption for system (2). Finally, the requirement on $f_{2}$ in item 5 , along with the condition in item 4 and the growth condition on $g_{2}$ in item 2 , simply guarantee that the set $\mathscr{A} \times\{0\}$, representing the final desired attractor for the state of (2), is forward invariant for (2) with $u \equiv 0$. We remark how the requirement on $f_{2}$ in item 5 is indeed necessary if the goal is to design continuous static state-feedback control laws.

Under the previous conditions it is possible to formulate the next theorem which is the main result of the paper.

Theorem 2

Under Assumption 1, there exists a continuous feedback law of the form

$$
u=-\operatorname{sgn}\left(x_{2}\right) \kappa\left(\left|x_{2}\right|\right) \psi(|x|)
$$

where $x=\operatorname{col}\left(x_{1}, x_{2}\right), \kappa \in \mathscr{K}_{\infty}$ and $\psi: \mathbb{R}_{\geqslant 0} \rightarrow \mathbb{R}_{\geqslant 0}$ is continuous, such that, for the closed-loop system (2), (4), the set $[0, N] \times \mathscr{A} \times\{0\}$ is globally asymptotically stable.

Proof

Using item 4 of Assumption 1 and recalling Theorem 1 , let $V_{1}: \mathbb{R} \times \mathbb{R}^{n} \rightarrow \mathbb{R}_{\geqslant 0}$ be a Lyapunov function for (3). In particular, $V_{1}$ is smooth and there exist class- $\mathscr{K}_{\infty}$ functions $\underline{\alpha}$ and $\bar{\alpha}$ such that

$$
\underline{\alpha}\left(\left|x_{1}\right|_{\mathscr{A}}\right) \leqslant V_{1}\left(\tau, x_{1}\right) \leqslant \bar{\alpha}\left(\left|x_{1}\right|_{\mathscr{A}}\right) \quad \forall\left(\tau, x_{1}\right) \in[0, N] \times \mathbb{R}^{n}
$$

and

$$
\begin{array}{rr}
\frac{\partial V_{1}\left(\tau, x_{1}\right)}{\partial \tau} \tau^{\prime}+\frac{\partial V_{1}\left(\tau, x_{1}\right)}{\partial x_{1}} f_{1}\left(\tau, x_{1}, 0\right) \leqslant-V_{1}\left(\tau, x_{1}\right) & \forall\left(\tau, x_{1}\right) \in[0, N] \times C \\
V_{1}\left(\tau-1, g_{1}\left(\tau, x_{1}, 0\right)\right) \leqslant \frac{1}{2} V_{1}\left(\tau, x_{1}\right) & \forall\left(\tau, x_{1}\right) \in[1, N] \times D
\end{array}
$$

for all $\tau^{\prime} \in[0, \delta]$. Using item 2 of Assumption 1 and the smoothness of $V_{1}$, there exist continuous, nondecreasing functions $\psi_{1}, \psi_{2}, \psi_{3}: \mathbb{R}_{\geqslant 0} \rightarrow \mathbb{R}_{\geqslant 0}$ such that, for all $\left(\tau, x_{1}, x_{2}\right) \in[1, N] \times D \times \mathbb{R}$,

$$
\begin{gathered}
\left|g_{1}\left(\tau, x_{1}, x_{2}\right)\right| \leqslant \psi_{1}(|x|) \\
\left|g_{2}\left(\tau, x_{1}, x_{2}\right)\right| \leqslant \psi_{2}(|x|)\left|x_{2}\right| \\
2\left[V_{1}\left(\tau-1, g_{1}\left(\tau, x_{1}, x_{2}\right)\right)-V_{1}\left(\tau-1, g_{1}\left(\tau, x_{1}, 0\right)\right)\right]^{2} \leqslant \psi_{3}(|x|)\left|x_{2}\right|^{2} .
\end{gathered}
$$

Let the smooth, class- $\mathscr{K}_{\infty}$ functions $\rho_{1, k}, \rho_{2, k}$ and positive constants $\mu_{k}$, where $k \in\{0, \ldots, 2 N\}$, satisfy

1. $1 \leqslant \mu_{0} \leqslant \mu_{1} \leqslant \cdots \leqslant \mu_{2 N}$,

2. for all $s \geqslant 0$,

$$
0 \leqslant \rho_{1,0}(s) \leqslant \rho_{1,1}(s) \leqslant \cdots \leqslant \rho_{1,2 N}(s)
$$

and

$$
0 \leqslant \rho_{2,0}(s) \leqslant \rho_{2,1}(s) \leqslant \cdots \leqslant \rho_{2,2 N}(s),
$$

3. for all $\left(x_{1}, x_{2}\right) \in D \times \mathbb{R}$, and $k \in\{0, \ldots, 2 N-1\}$,

$$
\begin{aligned}
& \psi_{3}(|x|)+\left[\mu_{k}+\rho_{1, k}\left(\psi_{1}(|x|)\right)+\rho_{2, k}\left(\psi_{2}(|x|)\left|x_{2}\right|\right)\right] \psi_{2}(|x|)^{2} \\
& \leqslant 0.5\left[\mu_{k+1}+\rho_{1, k+1}\left(\left|x_{1}\right|\right)+\rho_{2, k+1}\left(\left|x_{2}\right|\right)\right] .
\end{aligned}
$$


Let $\sigma:[0,1] \rightarrow[0,1]$ be a smooth, nondecreasing function that is zero on a neighborhood of zero and is one on a neighborhood of one. For $\left(\tau, x_{1}, x_{2}\right) \in[0, N] \times \mathbb{R}^{n} \times \mathbb{R}$, define

$$
\begin{aligned}
W\left(\tau, x_{1}, x_{2}\right):= & (1-\sigma(2 \tau-\lfloor 2 \tau\rfloor))\left(\mu_{\lfloor 2 \tau\rfloor}+\rho_{1,\lfloor 2 \tau\rfloor}\left(\left|x_{1}\right|\right)+\rho_{2,\lfloor 2 \tau\rfloor}\left(\left|x_{2}\right|\right)\right) \\
& +\sigma(2 \tau-\lfloor 2 \tau\rfloor)\left(\mu_{\lfloor 2 \tau\rfloor+1}+\rho_{1,\lfloor 2 \tau\rfloor+1}\left(\left|x_{1}\right|\right)+\rho_{2,\lfloor 2 \tau\rfloor+1}\left(\left|x_{2}\right|\right)\right)
\end{aligned}
$$

where, for each $\tau \in \mathbb{R},\lfloor 2 \tau\rfloor=k$ where $k$ is the unique integer satisfying $2 \tau \in[k, k+1)$. The definition of $W$ can be extended to $\mathbb{R} \times \mathbb{R}^{n} \times \mathbb{R}$ by projecting $\tau$ into the interval $[0, N]$. Observe that $W$ is smooth, nondecreasing in $\tau$, and $W\left(\tau, x_{1}, x_{2}\right) \geqslant 1$ for all $\left(\tau, x_{1}, x_{2}\right) \in \mathbb{R} \times \mathbb{R}^{n} \times \mathbb{R}$. Define

$$
V_{2}\left(\tau, x_{1}, x_{2}\right):=W\left(\tau, x_{1}, x_{2}\right)\left|x_{2}\right|^{2} .
$$

Note that

$$
\begin{aligned}
\frac{\partial V_{2}\left(\tau, x_{1}, x_{2}\right)}{\partial x_{2}}= & 2 W\left(\tau, x_{1}, x_{2}\right) x_{2}+\left[(1-\sigma(2 \tau-\lfloor 2 \tau\rfloor)) \rho_{\lfloor 2 \tau\rfloor}^{\prime}\left(\left|x_{2}\right|\right)\right. \\
& \left.+\sigma(2 \tau-\lfloor 2 \tau\rfloor) \rho_{2,\lfloor 2 \tau\rfloor+1}^{\prime}\left(\left|x_{2}\right|\right)\right] \operatorname{sgn}\left(x_{2}\right)\left|x_{2}\right|^{2} .
\end{aligned}
$$

In particular, for all $\left(\tau, x_{1}, x_{2}\right) \in[0, N] \times C \times \mathbb{R}$,

$$
\begin{aligned}
\operatorname{sgn}\left(x_{2}\right) & =\operatorname{sgn}\left(\left|\frac{\partial V_{2}\left(\tau, x_{1}, x_{2}\right)}{\partial x_{2}}\right|\right) \\
\left|x_{2}\right| & \leqslant\left|\frac{\partial V_{2}\left(\tau, x_{1}, x_{2}\right)}{\partial x_{2}}\right| \\
\left|\frac{\partial V_{2}\left(\tau, x_{1}, x_{2}\right)}{\partial x_{2}}\right| & \leqslant\left|x_{2}\right| \psi_{4}(|x|)
\end{aligned}
$$

for some continuous function $\psi_{4}$.

Consider the Lyapunov function

$$
V\left(\tau, x_{1}, x_{2}\right):=V_{1}\left(\tau, x_{1}\right)^{2}+V_{2}\left(\tau, x_{1}, x_{2}\right) .
$$

Then, using the second inequality of (6), the fact that $W\left(\cdot, x_{1}, x_{2}\right)$ is nondecreasing, the inequalities (7), the definition of $W$ in (11), and the relation (10), we have for all $\left(\tau, x_{1}, x_{2}\right) \in[1, N] \times D \times \mathbb{R}$,

$$
\begin{aligned}
& V\left(\tau-1, g_{1}\left(\tau, x_{1}, x_{2}\right), g_{2}\left(\tau, x_{1}, x_{2}\right)\right) \\
&=V_{1}\left(\tau-1, g_{1}\left(\tau, x_{1}, x_{2}\right)\right)^{2}+V_{2}\left(\tau-1, g_{1}\left(\tau, x_{1}, x_{2}\right), g_{2}\left(\tau, x_{1}, x_{2}\right)\right) \\
& \leqslant 2 V_{1}\left(\tau-1, g_{1}\left(\tau, x_{1}, 0\right)\right)^{2}+2\left(V_{1}\left(\tau-1, g_{1}\left(\tau, x_{1}, x_{2}\right)\right)-V_{1}\left(\tau-1, g_{1}\left(\tau, x_{1}, 0\right)\right)\right)^{2} \\
&+V_{2}\left(\tau-1, g_{1}\left(\tau, x_{1}, x_{2}\right), g_{2}\left(\tau, x_{1}, x_{2}\right)\right) \\
& \leqslant 0.5 V_{1}\left(\tau, x_{1}\right)^{2}+\psi_{3}(|x|)\left|x_{2}\right|^{2}+W\left(\tau-1, g_{1}\left(\tau, x_{1}, x_{2}\right), g_{2}\left(\tau, x_{1}, x_{2}\right)\right)\left|g_{2}\left(\tau, x_{1}, x_{2}\right)\right|^{2} \\
& \leqslant 0.5 V_{1}\left(\tau, x_{1}\right)^{2}+\psi_{3}(|x|)\left|x_{2}\right|^{2}+W\left(0.5\lfloor 2 \tau-1\rfloor, g_{1}\left(\tau, x_{1}, x_{2}\right), g_{2}\left(\tau, x_{1}, x_{2}\right)\right)\left|g_{2}\left(\tau, x_{1}, x_{2}\right)\right|^{2} \\
&= 0.5 V_{1}\left(\tau, x_{1}\right)^{2}+\psi_{3}(|x|)\left|x_{2}\right|^{2}+\left(\mu_{\lfloor 2 \tau-1\rfloor}+\rho_{1,\lfloor 2 \tau-1\rfloor}\left(\left|g_{1}\left(\tau, x_{1}, x_{2}\right)\right|\right)\right. \\
&\left.+\rho_{2,\lfloor 2 \tau-1\rfloor}\left(\left|g_{2}\left(\tau, x_{1}, x_{2}\right)\right|\right)\right)\left|g_{2}\left(\tau, x_{1}, x_{2}\right)\right|^{2} \\
& \leqslant 0.5 V_{1}\left(\tau, x_{1}\right)^{2}+\psi_{3}(|x|)\left|x_{2}\right|^{2}+\left(\mu_{\lfloor 2 \tau-1\rfloor}+\rho_{1,\lfloor 2 \tau-1\rfloor}\left(\psi_{1}(|x|)\right)\right. \\
&\left.+\rho_{2,\lfloor 2 \tau-1\rfloor}\left(\psi_{2}(|x|)\left|x_{2}\right|\right)\right) \psi_{2}(|x|)^{2}\left|x_{2}\right|^{2}
\end{aligned}
$$




$$
\begin{aligned}
& \leqslant 0.5 V_{1}\left(\tau, x_{1}\right)^{2}+0.5\left(\mu_{\lfloor 2 \tau\rfloor}+\rho_{1,\lfloor 2 \tau\rfloor}\left(\left|x_{1}\right|\right)+\rho_{2,\lfloor 2 \tau\rfloor}\left(\left|x_{1}\right|\right)\right)\left|x_{2}\right|^{2} \\
& =0.5 V_{1}\left(\tau, x_{1}\right)^{2}+0.5 W\left(\lfloor\tau\rfloor, x_{1}, x_{2}\right)\left|x_{2}\right|^{2} \\
& \leqslant 0.5 V_{1}\left(\tau, x_{1}\right)^{2}+0.5 W\left(\tau, x_{1}, x_{2}\right)\left|x_{2}\right|^{2} \\
& =0.5 V_{1}\left(\tau, x_{1}\right)^{2}+0.5 V_{2}\left(\tau, x_{1}, x_{2}\right) \\
& =0.5 V\left(\tau, x_{1}, x_{2}\right) .
\end{aligned}
$$

Also, there exists a continuous function $\psi_{2}$ such that, for all $\left(\tau, x_{1}, x_{2}\right) \in[0, N] \times C \times \mathbb{R}$,

$$
\begin{aligned}
\dot{V}:= & \frac{\partial V\left(\tau, x_{1}, x_{2}\right)}{\partial \tau} \dot{\tau}+\frac{\partial V\left(\tau, x_{1}, x_{2}\right)}{\partial x_{1}} f_{1}\left(\tau, x_{1}, x_{2}\right)+\frac{\partial V\left(\tau, x_{1}, x_{2}\right)}{\partial x_{2}}\left[f_{2}\left(\tau, x_{1}, x_{2}\right)+b\left(\tau, x_{1}, x_{2}\right) u\right] \\
\leqslant & -2 V_{1}\left(\tau, x_{1}\right)^{2}+2 V_{1}\left(\tau, x_{1}\right) \frac{\partial V_{1}\left(\tau, x_{1}\right)}{\partial x_{1}}\left(f_{1}\left(\tau, x_{1}, x_{2}\right)-f_{1}\left(\tau, x_{1}, 0\right)\right) \\
& +\psi_{2}(x)\left|x_{2}\right|^{2}+\frac{\partial V_{2}\left(\tau, x_{1}, x_{2}\right)}{\partial x_{2}}\left[f_{2}\left(\tau, x_{1}, x_{2}\right)+b\left(\tau, x_{1}, x_{2}\right) u\right] .
\end{aligned}
$$

Using item 5 of Assumption 1 and (5), let $\rho_{3}$ be a bounded class- $\mathscr{K}$ function and let $\rho_{4}: \mathbb{R}_{\geqslant 0} \rightarrow \mathbb{R}_{\geqslant 0}$ be an unbounded, continuous, nondecreasing function that is zero on a neighborhood of zero such that

$$
\left|f_{2}\left(\tau, x_{1}, 0\right)\right| \leqslant \rho_{3}\left(V_{1}\left(\tau, x_{1}\right)\right)+\rho_{4}\left(V_{1}\left(\tau, x_{1}\right)\right) .
$$

Let $\rho^{*} \geqslant \rho_{3}(s)$ for all $s \geqslant 0$. Then, for each $\varepsilon>0$, by considering the two cases $|v| \leqslant \varepsilon s^{2} / \rho^{*}$ and $|v| \geqslant \varepsilon s^{2} / \rho^{*}$, we have

$$
|v| \rho_{3}(s) \leqslant \varepsilon s^{2}+|v| \rho_{3}\left(\sqrt{|v| \rho^{*} / \varepsilon}\right)
$$

and, by multiplying and dividing by $s$ and completing squares,

$$
|v| \rho_{4}(s) \leqslant \varepsilon s^{2}+(4 \varepsilon)^{-1}|v|^{2} \rho_{4}^{2}(s) / s^{2} .
$$

Note that $\rho_{4}(s) / s$ is a continuous function of $s$ that is zero for small $s$. It follows that

$$
\left|\frac{\partial V_{2}\left(\tau, x_{1}, x_{2}\right)}{\partial x_{2}} f_{2}\left(\tau, x_{1}, 0\right)\right| \leqslant 2 \varepsilon V_{1}\left(\tau, x_{1}\right)^{2}+\left|\frac{\partial V_{2}\left(\tau, x_{1}, x_{2}\right)}{\partial x_{2}}\right| \rho_{5}\left(\left|x_{2}\right|\right) \psi_{5}\left(|x|, \varepsilon^{-1}\right)
$$

where $\psi_{5}$ is continuous and $\rho_{5} \in \mathscr{K}_{\infty}$. So, with appropriate choice for $\varepsilon$, using the continuity of $f_{2}$ and the first of (13), and by item 3 of Assumption 1, we have the existence of a continuous function $\psi_{6}$ and a class- $\mathscr{K}_{\infty}$ function $\rho_{6}$ such that

$$
\dot{V} \leqslant-V_{1}\left(\tau, x_{1}\right)^{2}+\left|\frac{\partial V_{2}\left(\tau, x_{1}, x_{2}\right)}{\partial x_{2}}\right|\left(\left(\left|x_{2}\right|+\rho_{6}\left(\left|x_{2}\right|\right)\right) \psi_{6}(|x|)-\underline{b}(|x|) \kappa\left(\left|x_{2}\right|\right) \psi(|x|)\right) .
$$

We then pick $\kappa(s)=s+\rho_{6}(s)$ and

$$
\psi(s)=\frac{1}{\underline{b}(s)} \max \left\{2 \psi_{6}(s), 2 \psi_{7}(s)\right\}
$$

where the continuous function $\psi_{7}$ satisfies $V_{2}\left(\tau, x_{1}, x_{2}\right) \leqslant \psi_{7}(|x|)\left|x_{2}\right|^{2}$. This results in

$$
\underline{b}(|x|) \kappa\left(\left|x_{2}\right|\right) \psi(|x|) \geqslant\left(\left|x_{2}\right|+\rho_{6}\left(\left|x_{2}\right|\right)\right) \psi_{6}(|x|)+\left|x_{2}\right| \psi_{7}(|x|)
$$


and, in turn, by using the second of (13),

$$
\dot{V} \leqslant-V_{1}\left(\tau, x_{1}\right)^{2}-V_{2}\left(\tau, x_{1}, x_{2}\right)=-V\left(\tau, x_{1}, x_{2}\right) .
$$

This establishes the result.

In case just stabilization on compact sets is sought, the state feedback control law (4) reduces to a pure output feedback as detailed in the following corollary, which extends to a hybrid setting a similar result of [10].

\section{Corollary 1}

Under Assumption 1, there exists a continuous feedback law of the form

$$
u=-\operatorname{sgn}\left(x_{2}\right) \kappa\left(\left|x_{2}\right|\right) k,
$$

where $\kappa \in \mathscr{K}_{\infty}$ and $k>0$, such that, for the closed-loop system (2), (4), the set $[0, N] \times \mathscr{A} \times\{0\}$ is semi-globally asymptotically stable in the control parameter $k$. Namely, for any compact set $K \subset \mathbb{R}^{n} \times \mathbb{R}$, there exists a $k^{\star}>0$ such that for all $k \geqslant k^{\star}$ the set $[0, N] \times \mathscr{A} \times\{0\}$ is asymptotically stable for the closed-loop system (2), (17) with domain of attraction of the form $\mathbb{R} \times \mathscr{D}$ with $\mathscr{D} \supset K$.

Proof

The proof is the same as the one of Theorem 2 until Equation (15) which, by choosing the control as in (17), becomes

$$
\dot{V} \leqslant-V_{1}\left(\tau, x_{1}\right)^{2}+\left|\frac{\partial V_{2}\left(\tau, x_{1}, x_{2}\right)}{\partial x_{2}}\right|\left(\left(\left|x_{2}\right|+\rho_{6}\left(\left|x_{2}\right|\right)\right) \psi_{6}(|x|)-\underline{b}(|x|) \kappa\left(\left|x_{2}\right|\right) k\right) .
$$

Let $c>0$ be such that the compact set

$$
\Omega_{c}:=\left\{\left(\tau, x_{1}, x_{2}\right) \in[0, N] \times \mathbb{R}^{n} \times \mathbb{R}: V\left(\tau, x_{1}, x_{2}\right) \leqslant c\right\}
$$

satisfies $K \subseteq \Omega_{c}$, and let $b^{\star}:=\min _{x \in \Omega_{c}} \underline{b}(|x|), \psi_{6}^{\star}:=\max _{x \in \Omega_{c}} \psi_{6}(|x|)$ and $\psi_{7}^{\star}:=\max _{x \in \Omega_{c}} \psi_{7}(|x|)$. Then, by taking $k^{\star}=\left(1 / b^{\star}\right) \max \left\{\psi_{6}^{\star}, \psi_{7}^{\star}\right\}$, it follows that for all $k \geqslant k^{\star}$ and for any $\left(\tau, x_{1}, x_{2}\right) \in \Omega_{c}$ the bound (16) holds. From this the result follows.

\section{EXAMPLES}

\subsection{First example: suppressing the effect of discontinuous disturbances by state feedback}

As first example we consider a problem of disturbance suppression for a particular class of hybrid systems. Specifically, we consider the control system

$$
\left.\begin{array}{l}
\dot{\sigma}=1 \\
\dot{w}=0 \\
\dot{\eta}=F \eta+G w+r(w, \eta, y) y \\
\dot{y}=f(w, \eta, y)+b(w, \eta, y) u
\end{array}\right\} \quad(\sigma, w, \eta, y) \in[0, \pi] \times[-10,10] \times \mathbb{R}^{2} \times \mathbb{R}
$$

in which $(F, G) \in \mathbb{R}^{2 \times 2} \times \mathbb{R}^{2 \times 1}$ is a controllable pair with $F$ Hurwitz and $r, f$ and $b$ are continuous functions with $b(w, \eta, y) \geqslant \underline{b}>0$. What makes hybrid the previous system is the presence of the 
variable $w$, playing the role of disturbance, which jumps between two constant values $\left\{-w^{\star}, w^{\star}\right\}$, with $w^{\star} \in[-10,10]$, with a periodicity of $\pi$ governed by the clock variable $\sigma$. For illustrative purposes, we focus on the following values of the pair $(F, G)$

$$
F=\left(\begin{array}{cc}
-2 & 1 \\
0 & -1
\end{array}\right) \quad G=\left(\begin{array}{l}
0 \\
1
\end{array}\right)
$$

and we will restrict the possible $f$ and $b$ to the class of continuous functions satisfying $f(w, \eta, 0)=$ $w$ and $b(w, \eta, 0)=1$. First, observe that, by taking $N \geqslant 1$ and $\delta \geqslant 1 / \pi$, every solution of (19) can be generated as a solution of the system

$$
\left.\left.\begin{array}{l}
\dot{\tau} \in[0, \delta] \\
\dot{\sigma}=1 \\
\dot{w}=0 \\
\dot{\eta}=F \eta+G w+r(w, \eta, y) y \\
\dot{y}=f(w, \eta, y)+b(w, \eta, y) u
\end{array}\right\} \quad \begin{array}{l}
(\tau, \sigma, w, \eta, y) \in[0, N] \times[0, \pi] \times[-10,10] \times \mathbb{R}^{2} \times \mathbb{R} \\
\tau^{+}=\tau-1 \\
\sigma^{+}=0 \\
w^{+}=-w \\
\eta^{+}=\eta \\
y^{+}=y
\end{array}\right\} \quad(\tau, \sigma, w, \eta, y) \in[1, N] \times\{\pi\} \times[-10,10] \times \mathbb{R}^{2} \times \mathbb{R}
$$

with $\tau(0,0)=N$. As a consequence, system (19) can be cast in the form (2).

We focus now on the zero dynamics of (19), namely on system

$$
\left.\begin{array}{l}
\dot{\tau} \in[0, \delta] \\
\dot{\sigma}=1 \\
\dot{w}=0 \\
\dot{\eta}=F \eta+G w
\end{array}\right\} \quad(\tau, \sigma, w, \eta, y) \in[0, N] \times[0, \pi] \times[-10,10] \times \mathbb{R}^{2}
$$

Using the fact that $F$ is Hurwitz and by defining

$$
\Pi(\sigma)=\left[\begin{array}{c}
\frac{1}{2}-\frac{2 \exp (-\sigma)}{1+\exp (-\pi)}+\frac{\exp (-2 \sigma)}{1+\exp (-2 \pi)} \\
1-\frac{2 \exp (-\sigma)}{1+\exp (-\pi)}
\end{array}\right]
$$

it can be verified that there exist $\mu>0$ and $\lambda>0$ such that each solution $x=(\tau, \sigma, w, \eta)$ of (21) satisfies

$$
|\eta(t, j)-\Pi(\sigma(t, j)) w(t, j)| \leqslant \mu \exp (-\lambda(t+j))|\eta(0,0)-\Pi(\sigma(0,0)) w(0,0)|
$$


for all $(t, j) \in \operatorname{dom} x$. Thus, we conclude that the system (21) has the compact set $[0, N] \times \mathscr{A}$ globally asymptotically stable where

$$
\mathscr{A}:=\left\{(\sigma, w, \eta) \in \times[0, \pi] \times[-10,10] \times \mathbb{R}^{2}: \eta=\Pi(\sigma) w\right\} .
$$

Furthermore, it is not difficult to verify that $|\Pi(\sigma)|>0$ for all $\sigma \in[0, \pi]$. Indeed, in order to have $\Pi(\sigma)=0, \sigma \in[0, \pi]$ must satisfy

$$
\frac{2 \exp (-\sigma)}{1+\exp (-\pi)}=\frac{2 \exp (-2 \sigma)}{1+\exp (-2 \pi)}=1
$$

which is impossible. With these results at hand, we preliminarily choose the control law as

$$
u=-\frac{(\Pi(\sigma))^{\mathrm{T}}}{|\Pi(\sigma)|^{2}} \eta+v
$$

where $v$ is a residual control input that is used to compensate for the effect of the disturbance $w$ on the output dynamics when the zero dynamics belong to the set $[0, N] \times \mathscr{A}$ and $y$ is zero. This choice guarantees that, by defining

$$
f_{2}(\tau, \sigma, w, \eta, y):=f(w, \eta, y)-b(w, \eta, y)\left(\frac{\Pi(\sigma)}{|\Pi(\sigma)|^{2}}\right)^{\mathrm{T}} \eta
$$

and by bearing in mind that $f(w, \eta, 0)=w$ and $b(w, \eta, 0)=1, f_{2}(\tau, \sigma, w, \eta, 0)=0$ for all $(\tau, \sigma, w, \eta) \in[0, N] \times \mathscr{A}$. Therefore, all the conditions of Assumption 1 hold. It follows from Corollary 1 that, for the system (19), the set $\mathscr{A} \times\{0\}$ is semiglobally asymptotically stabilizable by a feedback $v$ of the form $v=-\operatorname{sgn}(y) \kappa(|y|) k$ where $k>0$.

The previous example draws its inspiration from problems of hybrid output regulation, currently under study by the authors, with the proposed solution that can be interpreted according to internal model-based design strategies (see [10,19]). Specifically, with an eye to (26), the first term of the control law, dependent on $(\sigma, \eta)$, can be interpreted as the term aiming to compensate for the non vanishing steady-state value of $f(w, \eta, y)$, by thus guaranteeing that the system has a steady-state solution, characterized by $y=0$, with $v=0$. On the other hand, the second term of (26), dependent on $y$ through the high-gain law, can be interpreted as a stabilization term having the role of steering the state of the overall system towards the desired steady-state. Under this perspective, the state variable $\eta$ plays the role of an 'internal-model' state variable, most likely part of the control dynamics rather than of the controlled plant. In order to give the reader more details about the previous interpretation, consider the controlled system

$$
\begin{aligned}
& \left.\begin{array}{rl}
\dot{\sigma} & =1 \\
\dot{w} & =0 \\
\dot{y} & =f(w, y)+u
\end{array}\right\} \quad(\sigma, w, y) \in[0, \pi] \times[-10,10] \times \mathbb{R} \\
& \left.\begin{array}{rl}
\sigma^{+} & =0 \\
w^{+} & =-w \\
y^{+} & =y
\end{array}\right\} \quad(\sigma, w, y) \in\{\pi\} \times[-10,10] \times \mathbb{R}
\end{aligned}
$$

with $f(w, 0)=w$, where $w$ is a jumping disturbance, and the problem of steering the output $y$ to zero by means of a feedback processing $\sigma$ and $y$ only. In this scenario, the controller is required to have the ability to compensate for the term $f(w, y)$, not vanishing at the desired steady-state, by providing the (jumping) steady-state control input $u=-f(w, 0)=-w$. By drawing inspiration from the theory of output regulation for continuous-time systems presented in [19] (see also [20]), we consider a hybrid dynamic controller flowing according to the dynamics

$$
\dot{\xi}=F \xi-G u,
$$


with $(F, G) \in \mathbb{R}^{2 \times 2} \times \mathbb{R}^{2 \times 1}$ the controllable pair specified above, whenever system (28) flows, and jumping, according to the trivial rule $\xi^{+}=\xi$, whenever system (28) experiences a jump, and the control law

$$
u=-\frac{(\Pi(\sigma))^{\mathrm{T}}}{|\Pi(\sigma)|^{2}} \xi+v
$$

where $v$ is a residual control input. After the change of variables $\xi \mapsto \eta=\xi+G y$, it turns out that the overall closed-loop system reads as (19) with the $\dot{y}$ equation replaced by

$$
\dot{y}=f_{2}(w, \eta, y)+v
$$

where $f_{2}(w, \eta, y)=f(w, y)-(\Pi(\sigma))^{\mathrm{T}} /|\Pi(\sigma)|^{2}(\eta-G y)$ and $r(w, \eta, y)$ such that $r(w, \eta, y) y=$ $G f(w, y)-G w-F G y$. By construction, it turns out that $f_{2}(w, \Pi(\sigma) w, 0)=0$ and, a consequence, the system fits in the framework of the previous section with the set $\mathscr{A} \times\{0\}$ that can be semiglobally asymptotically stabilized by a feedback of the form $v=-\operatorname{sgn}(y) \kappa(|y|) k$ where $k>0$.

\subsection{Second example: stabilization in presence of switching zero-dynamics with average} dwell-time

We consider a system of the form

$$
\begin{aligned}
& \dot{z}=A_{q} z+B_{q} y \\
& \dot{y}=f(z, y, q)+u
\end{aligned}
$$

with input $u \in \mathbb{R}$ and output $y \in \mathbb{R}$, in which $q$ is a discrete variable taking values in the set $Q:=\{-1,1\}$. The matrices $A_{-1}$ and $A_{1}$ are Hurwitz matrices while the function $f$ is continuous and such that $f(0,0, Q)=0$.

In the specific example, the rule underlying the switching between the matrices $A_{1}, A_{-1}$ is governed by the zero crossing of a continuous-time quasi-periodic signal of the form $\xi_{1}(t)+v_{1}(t)$ generated by the autonomous system

$$
\begin{aligned}
& \dot{\xi}_{1}=\Omega_{s} \xi_{2} \\
& \dot{\xi}_{2}=-\Omega_{s} \xi_{1} \\
& \dot{v}_{1}=\Omega_{n} v_{2} \\
& \dot{v}_{2}=-\Omega_{n} v_{1}
\end{aligned}
$$

whose initial conditions are assumed to range in the (invariant) set $\Xi \times V$ where

$$
\Xi:=\left\{\xi \in \mathbb{R}^{2}:|\xi|=\bar{\xi}\right\} \quad V:=\left\{v \in \mathbb{R}^{2}:|v| \in[0, \bar{v}]\right\}
$$

with $\bar{v}<\bar{\xi}$ positive numbers. The signal $\xi_{1}(t)+v_{1}(t)$ can be conveniently interpreted as the superimposition of a deterministic clock signal $\xi_{1}(t)$, playing the role of temporization signal, and of a high-frequency signal $v_{1}(t)$, playing the role of additive noise. In this respect we suppose that $\Omega_{n} \gg \Omega_{s}$ and that $\bar{\xi} \gg \bar{v}$. Furthermore, while $\bar{\xi}$ and $\Omega_{s}$ will be regarded as fixed parameters, the stability conditions presented in the following will be given in terms of the upper bound $\bar{v}$ of the noise amplitude which, in turn, will be regarded as a function of the frequency $\Omega_{n}$. Specifically, by restricting the attention to physical signals $v_{1}(t)$ having finite energy, we will assume that $\bar{v}$ is a decreasing function of $\Omega_{n}$ fulfilling the bound

$$
\bar{v}\left(\Omega_{n}\right) \leqslant \frac{P}{\Omega_{n}}
$$

where $P$ is a positive constant. 
The switching rule for $q$ is taken of the form $q^{+}=-q$ if the signal $\xi_{1}(t)+v_{1}(t)$ has a zero crossing and its time derivative has a sign which is opposite to the sign of $q$, while $q$ is kept constant, namely $\dot{q}=0$, otherwise. Formally,

$$
(\xi, v) \in C_{q}:=\left\{(\xi, v) \in \Xi \times V: q\left(\xi_{1}+v_{1}\right) \geqslant 0\right\} \Rightarrow \dot{q}=0
$$

and

$$
(\xi, v) \in D_{q}:=\left\{(\xi, v) \in \Xi \times V: \xi_{1}+v_{1}=0, q\left(\xi_{2}+v_{2}\right) \leqslant 0\right\} \Rightarrow q^{+}=-q .
$$

By assuming continuity of the state $(z, y)$ during jumps, the overall dynamics can thus be written as the hybrid system

$$
\left.\begin{array}{rl}
\dot{z} & =A_{q} z+B_{q} y \\
\dot{q} & =0 \\
\dot{\xi}_{1} & =\Omega_{s} \xi_{2} \\
\dot{\xi}_{2} & =-\Omega_{s} \xi_{1} \\
\dot{v}_{1} & =\Omega_{n} v_{2} \\
\dot{v}_{2} & =-\Omega_{n} v_{1} \\
\dot{y} & =f(z, y, q)+u
\end{array}\right\} \quad(z,(q, \xi, v), y) \in \mathbb{R}^{2} \times\left(\bigcup_{q \in Q}\left(\{q\} \times C_{q}\right)\right) \times \mathbb{R}
$$

It turns out that the time interval between two consecutive switches of the previous system satisfies an average dwell-time depending on the specified data. As a matter of fact note that there exist time intervals $\left\{t:\left|\xi_{1}(t)\right| \leqslant \bar{v}\right\}$, centered at the times at which $\xi_{1}(t)$ crosses the zero and regularly spaced by $\pi / \Omega_{s}$, within which the signal $\xi_{1}(t)+v_{1}(t)$ might have a number of zero crossing, due to the superimposed high-frequency noise, and outside which switches do not occur. A simple computation shows that an estimation of the length $L$ of each of these time intervals is a function of $\bar{v}\left(\Omega_{n}\right)$ and it is given by

$$
L\left(\bar{v}\left(\Omega_{n}\right)\right)=\frac{2}{\Omega_{s}} \arctan \frac{\bar{v}\left(\Omega_{n}\right)}{\sqrt{\bar{\xi}^{2}-\bar{v}^{2}\left(\Omega_{n}\right)}} .
$$

Note that $L \rightarrow 0$ as $\Omega_{n} \rightarrow \infty$. Within these intervals an estimation $N_{s}$ of the number of times the noisy signal $\xi_{1}(t)+v_{1}(t)$ changes sign (and thus an upper bound of the times $q$ resets) is a function of $\bar{v}\left(\Omega_{n}\right)$ and of $\Omega_{n}$ given by

$$
N_{s}\left(\bar{v}\left(\Omega_{n}\right), \Omega_{n}\right)=L\left(\bar{v}\left(\Omega_{n}\right)\right) \frac{\Omega_{n}}{\pi} .
$$

By taking advantage from (29), it turns out that there exists a $N>0$ such that $N_{s}\left(\bar{v}\left(\Omega_{n}\right), \Omega_{n}\right) \leqslant N$ for all $\Omega_{n}>0$ and $\lim _{\Omega_{n} \rightarrow \infty} N_{s}\left(\bar{v}\left(\Omega_{n}\right), \Omega_{n}\right) \leqslant 2 P / \Omega_{s} \bar{\xi}$.

As a consequence, for each solution $x$ and each $(s, i),(t, j) \in \operatorname{dom} x$ of (30) with $s+i \leqslant t+j$, the following estimation can be derived

$$
i-j \leqslant N+\delta(t-s) \quad \text { where } \delta=\frac{\Omega_{s}}{\pi} N_{s}\left(\bar{v}\left(\Omega_{s}\right), \Omega_{n}\right)
$$


Note that, by using (29), for any $\delta^{\star}>0$ there exists a $P^{\star}$ such that $\delta \leqslant \delta^{\star}$ for any $P \leqslant P^{\star}$ and for all $\Omega_{n} \geqslant 0$. By letting $x_{1}:=\operatorname{col}(z, q, \xi, v), x_{2}:=y$,

$$
C:=\mathbb{R}^{2} \times\left(\bigcup_{q \in Q}\left(\{q\} \times C_{q}\right)\right), \quad D:=\mathbb{R}^{2} \times\left(\bigcup_{q \in Q}\left(\{q\} \times C_{q}\right)\right)
$$

every solution of (30) can thus be generated as a solution of the system (2).

Now we check Assumption 1 and specifically item 4. The zero dynamics of the system is of the form

$$
\left.\begin{array}{l}
\dot{\tau}=[0, \delta] \\
\dot{z}=A_{q} z \\
\dot{q}=0 \\
\dot{\xi}_{1}=\Omega_{s} \xi_{2} \\
\dot{\xi}_{2}=-\Omega_{s} \xi_{1} \\
\dot{v}_{1}=\Omega_{n} v_{2} \\
\dot{v}_{2}=-\Omega_{n} v_{1}
\end{array}\right\} \quad(\tau, z,(q, \xi, v)) \in[0, N] \times \mathbb{R}^{2} \times\left(\bigcup_{q \in Q}\left(\{q\} \times C_{q}\right)\right)
$$

By using Theorem 2 in [11], it turns out that there exists a $\delta^{\star}$ such that the switching system $\dot{z}=A_{q(t)} z$, with $q(t)$ any possible switching sequence generated by (31) with $\delta \leqslant \delta^{\star}$, is uniformly exponentially stable. As a consequence there exists a $P^{\star}>0$ such that for any $P \leqslant P^{\star}$ the set $[0, N] \times \mathscr{A}$ with $\mathscr{A}=\{0\} \times Q \times \Xi \times V$ is globally asymptotically stable for (31). Therefore, all of the conditions of Assumption 1 hold. It follows from Corollary 1 that, for the system (2), the set $[0, N] \times \mathscr{A} \times\{0\}$ is semi-globally asymptotically stabilizable by a feedback $u$ of the form $u=-\operatorname{sgn}(y) \kappa(|y|) k$ where $k>0$.

\section{CONCLUSIONS}

The work focused on the global stabilization of the class of hybrid systems described by (2) under a minimum-phase assumption, which requires the existence of a compact set which is asymptotically stable for the reduced dynamics given by (3). Both global state-feedback and semi-global outputfeedback results have been given in Section 3. The structure of (2), under the conditions listed in Assumption 1, can be considered as the natural hybrid version of continuous-time systems in normal form having unitary relative degree and globally asymptotically stable zero dynamics. In this respect, the contribution of the work can be seen as the generalization of fundamental stabilization tools, both state- and output-feedback, proposed in the literature of nonlinear continuous-time systems in the past. The results presented in this paper represent a first attempt of the authors towards the development of a general theory of output regulation for linear and nonlinear hybrid systems. Specifically, the future attempt will be directed to extend the theory of nonlinear output regulation proposed in the continuous-time setting in [20] (see also [19]) to a general hybrid setting. In this respect the tools proposed in Theorem 2 and Corollary 1 will represent a building block for the design of internal-model based regulators for hybrid systems. 


\section{REFERENCES}

1. Liberzon D, Morse AS. Basic problems in stability and design of switched systems. IEEE Control Systems Magazine 1999; 19:59-70.

2. Liberzon D. Switching in Systems and Control. Birkhaüser: Cambridge, MA, 2003.

3. Branicky MS, Borkar VS, Mitter SK. A unified framework for hybrid control: model and optimal control theory. IEEE Transaction on Automatic Control 1998; 43(1):31-45.

4. Chen T, Francis B. Optimal Sampled-Data Control Systems. Springer: London, 1995.

5. Åström KJ. Event based control. In Analysis and Design of Nonlinear Control Systems, Astolfi A, Marconi L (eds). Springer: New York, 2008; 127-147.

6. Goebel R, Sanfelice R, Teel AR. Hybrid dynamics systems. IEEE Control System Magazine 2009; 29:28-93.

7. Byrnes C, Isidori A. Asymptotic stabilization of minimum phase nonlinear systems. IEEE Transaction on Automatic Control 1991; 36(10):1122-1137.

8. Byrnes CI, Isidori A. A frequency domain philosophy for nonlinear systems, with application to stabilization and to adaptive control. Proceedings of the 23rd Conference on Decision and Control, Las Vegas, NV, 12-14 December 1984.

9. Byrnes C, Isidori A. Local stabilization of minimum phase nonlinear systems. Systems and Control Letters 1988; 11:9-17.

10. Teel AR, Praly L. Tools for semiglobal stabilization by partial state and output feedback. SIAM Journal on Control and Optimization 1995; 33:1443-1485.

11. Hespanha JP, Morse AS. Stability of switched systems with average dwell-time. Proceedings of the 38th Conference on Decision and Control, Phoenix, Arizona, 1999.

12. Morse AS. Supervisory control of families of linear set-point controllers—part I: exact matching. IEEE Transaction on Automatic Control 1996; 41(10):1413-1431.

13. Zhai G, Hu B, Yasuka K, Michel AN. Stability analysis of switched systems with stable and unstable subsystems: an average dwell time approach. Proceedings of the American Control Conference, Chicago, 2000.

14. Hespanha JP, Liberzon D, Teel AR. On input-to-state stability of impulsive systems. Proceedings of the 44th Conference on Decision and Control, Seville, Spain, 2005.

15. Bacciotti A, Mazzi L. Remarks on dwell time solutions and stability of families of nonlinear vector fields. IEEE Transaction on Automatic Control 2009; 54(8):1886-1892.

16. Cai C, Teel AR, Goebel R. Smooth Lyapunov functions for hybrid systems, part II: (Pre-)asymptotically stable compact sets. IEEE Transaction on Automatic Control 2008; 53(3):734-748.

17. Goebel R, Teel AR. Solutions to hybrid inclusions via set and graphical convergence with stability theory applications. Automatica 2006; 42(4):573-587.

18. Isidori A. Nonlinear Control Systems II. Springer: New York, 1999.

19. Marconi L, Praly L, Isidori A. Output stabilization via nonlinear Luenberger observers. SIAM Journal on Control and Optimization 2007; 45(6):2277-2298.

20. Marconi L, Praly L. Uniform practical output regulation. IEEE Transaction on Automatic Control 2008; 53(5):1184-1202. 